\title{
Condition Monitoring of Rolling Bearings using Statistical Linguistic Analysis
}

\author{
Jinshan $\operatorname{Lin}^{1, a^{*}}$, Chunhong Dou ${ }^{2, b}$ \\ ${ }^{1}$ School of Mechatronics and Vehical Engineering, No. 5147 Dong Feng Dong Street, Weifang, \\ China \\ ${ }^{2}$ School of Information and Control Engineering, No. 5147 Dong Feng Dong Street, Weifang, China \\ ajslinmec@163.com, ${ }^{b}$ chdoucon@hotmail.com
}

Keywords: rolling bearing, condition monitoring, statistical linguistic analysis, rank index

Abstract. Defective rolling bearings generally provoke a demonstration of nonstationary and nonlinear properties. As a result, condition monitoring of a rolling bearing seems challenging due to difficulties in fault feature extraction. This study introduces statistical linguistic analysis (SLA) to investigate rolling bearing vibration data. By SLA, original vibration data are allowed to be distilled into a rank index sequence, which preserves fundamental dynamics hidden in the original data. Afterwards, a correlation coefficient is defined for detecting a change of conditions of rolling bearings. Consequently, this study develops a novel method for condition monitoring or rolling bearings using SLA. Moreover, the feasibility of the proposed method is assessed by using a set of full-lifecycle vibration data from a realistic rolling bearing. The results showed that the proposed method has the capability of detecting a change of running conditions of rolling bearings.

\section{Introduction}

Rolling bearings have illustrated wide application in diverse types of transmission of power. Accordingly, they play important role in industrial production. Once a rolling bearing breaks down, a normal process of production will be disturbed and an economic loss will be suffered. Therefore, it is a purposeful work to develop methods for condition monitoring of rolling bearings. However, vibration data collected from rolling bearings are usually polluted by noise. As a consequence, it is a hard problem to extract weak fault features from nonstationary, nonlinear and noisy data. Traditional techniques for vibration data analysis, based on linear and stationary theories, are hardly employed to explore vibration data from a defective rolling bearing. Currently, some time-frequency analysis (TFA) algorithms, such as wavelet transform [1, 2] and empirical mode decomposition [3, 4], have been presented for probing nonstationary and nonlinear data. Unfortunately, each of these TFA algorithms typically suffers from some deficiencies and leaves something to be desired. In this paper, statistical linguistic analysis (SLA) [5], a novel method for nonlinear data analysis, was pioneered in investigation of bearing vibration data. By SLA, original bearing vibration data are transformed into a rank index sequence. Furthermore, a correlation coefficient is defined for detecting a change of bearing running conditions. Thus, a novel method for condition monitoring of rolling bearings was proposed using SLA in this paper. Subsequently, the performance of the proposed method was evaluated by using realistic bearing vibration data containing different running stages of bearings. The results indicated that the proposed method can detect a change of running conditions of rolling bearings.

\section{The SLA-based method for condition monitoring}

Given a time series $x_{k}(k=1,2, \ldots, N)$, the SLA-based method is stated as follows.

(1) Translate the original sequence $x_{i}$ into a binary symbolic sequence

$b_{k}=\left\{\begin{array}{lll}0 & x_{k} \geq x_{k+1} & k=1,2, \cdots, N-1 \\ 1 & x_{k}<x_{k+1} & k=1,2, \cdots, N-1\end{array}\right.$ 
(2) Define $m$ successive characters as a word; by moving one data point each time, the original sequence $x_{k}$ is projected to a word collection with different word types; by counting the occurrence of each word type, the original sequence $x_{k}$ is again mapped to a rank index sequence with the length $2^{m}$.

(3) Compute the correlation coefficient between the rank index sequence for the starting moment of the running process and that for another moment; employ the correlation coefficient as a measure for detecting a change of running conditions of rolling bearings.

\section{Experimental verification}

In this section, the feasibility of the proposed method was tested by using full-lifecycle vibration data of a realistic rolling bearing [6]. These rolling bearing vibration data are depicted in Fig. 1. To begin with, two temporal statistical parameters, that is, Standard Deviation and Root Mean Square (RMS), served to monitor conditions of the rolling bearing and the results are demonstrated in Fig. 2 and 3, respectively. As shown in Fig. 2 and 3, the two temporal statistical parameters can detect a change of operating conditions of the rolling bearing at the moment of about 117 hours. Next, the proposed method was adopted for detecting a change of operating conditions of the rolling bearing. Probability distribution of the rank index is revealed in Fig. 4. As revealed in Fig. 4, the probability distribution of the rank index sequence exhibits power-law properties, especially in small time scales. This means that the rank index sequence describes natural characteristics of dynamics of the rolling bearing. Afterwards, the correlation coefficient between the rank index sequence for the beginning moment and that for another moment is displayed in Fig. 5. As displayed in Fig. 5, the proposed method can detect a change of bearing running conditions at the moment of about 89.17 hours, which is earlier than those acquired by the other two methods. The comparison between the proposed method and the two conventional methods suggested that the proposed method has a clear advantage over the traditional methods.

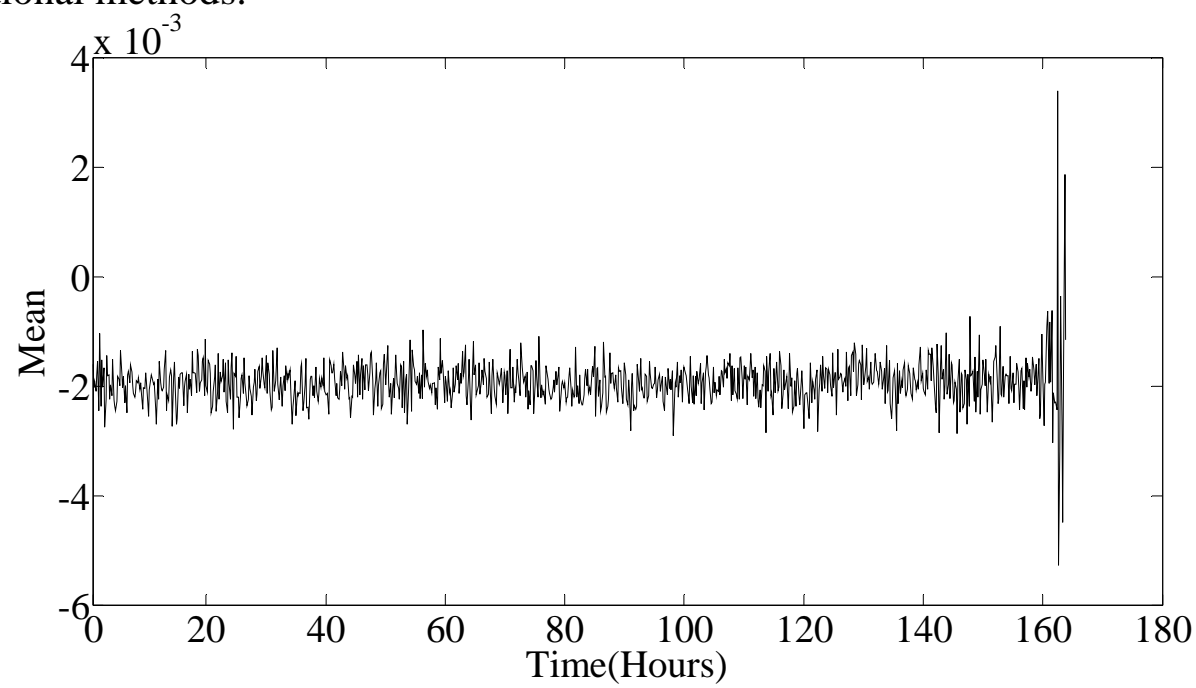

Fig. 1 The full-lifecycle vibration data of rolling bearings 


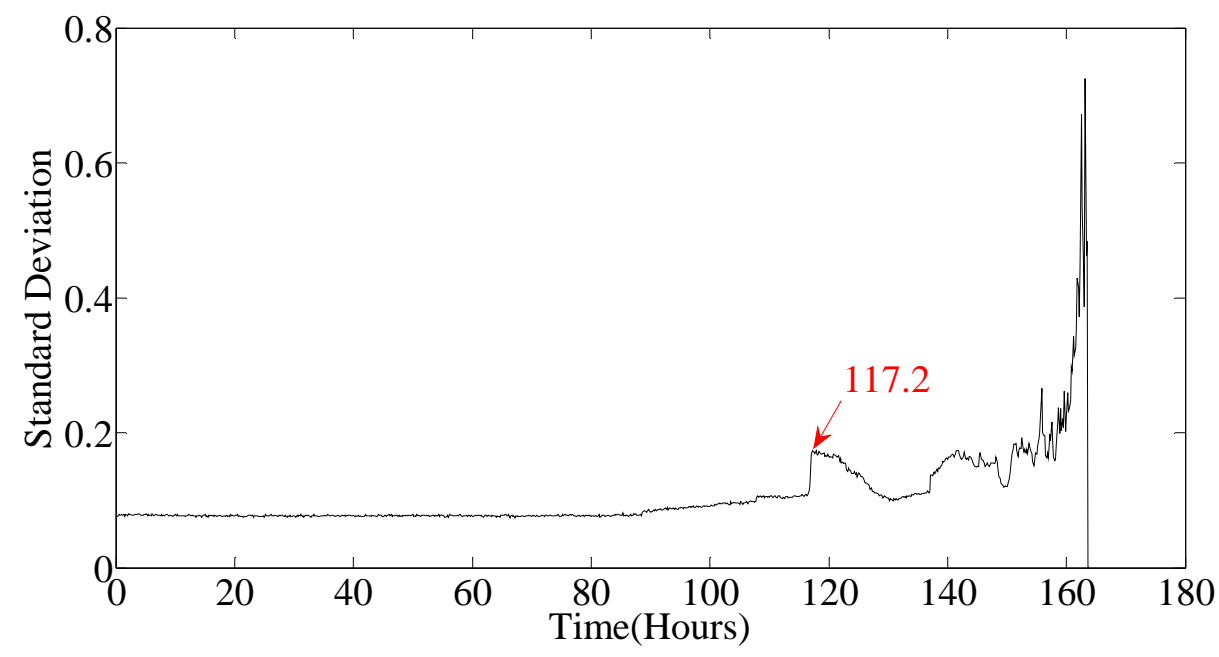

Fig. 2 Monitoring results for the rolling bearing using Standard Deviation

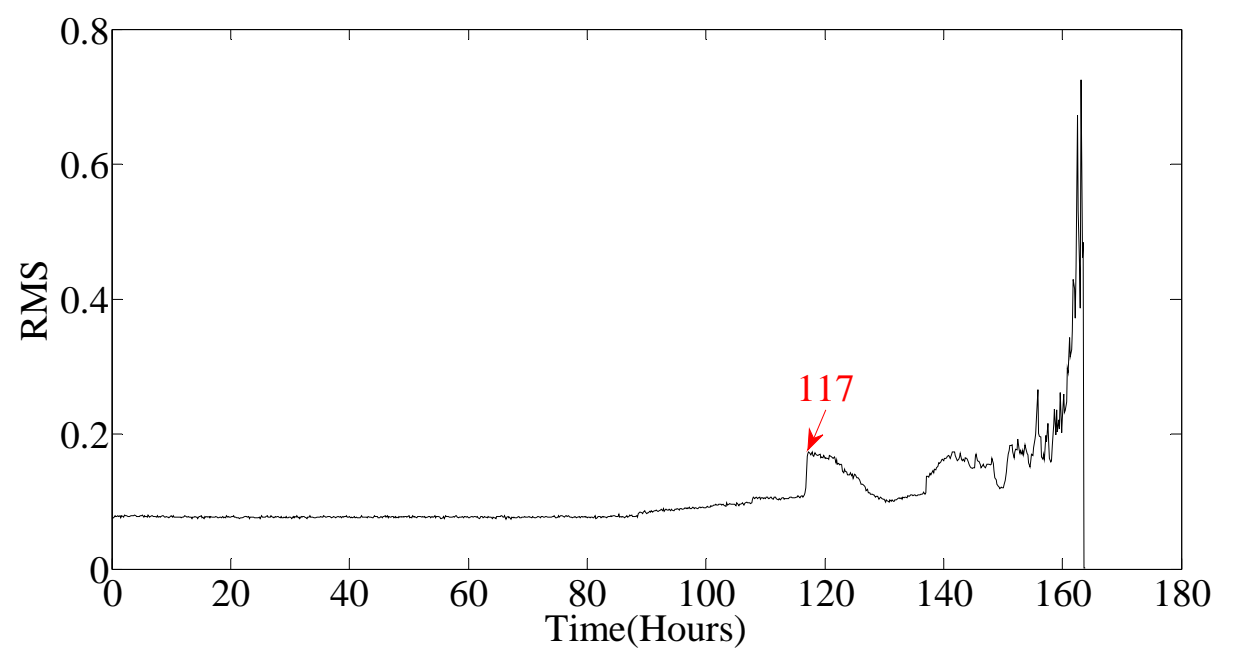

Fig. 3 Monitoring results for the rolling bearing using RMS

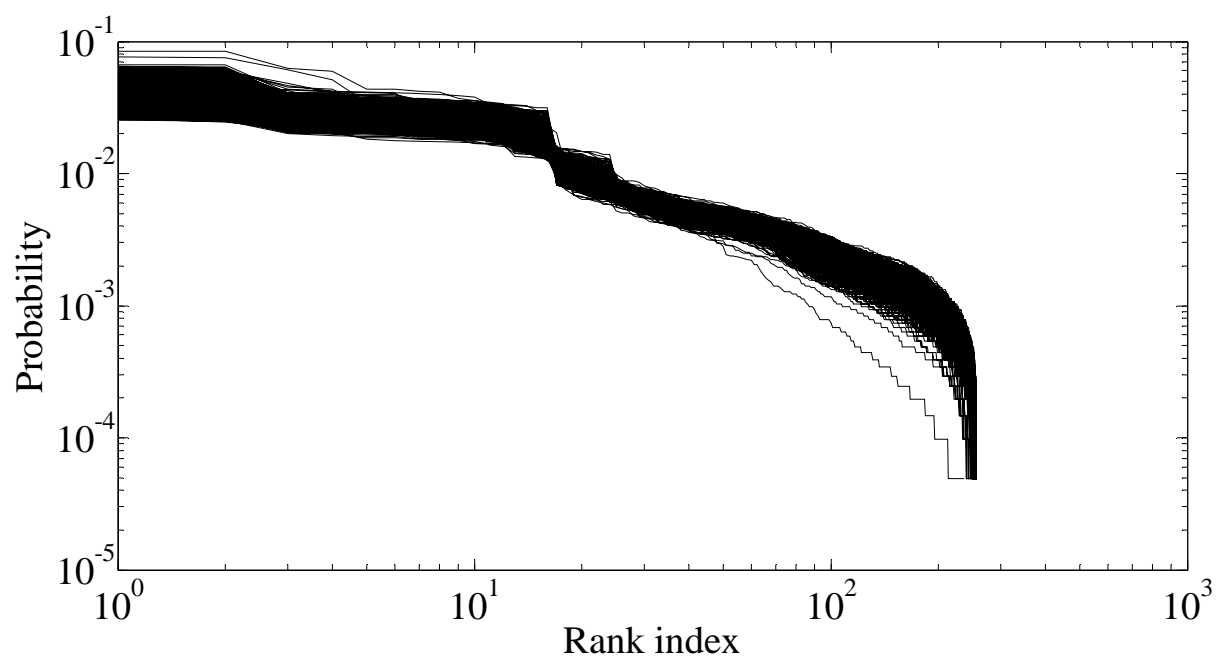

Fig. 4 Probability distribution of the rank index for the rolling bearing 


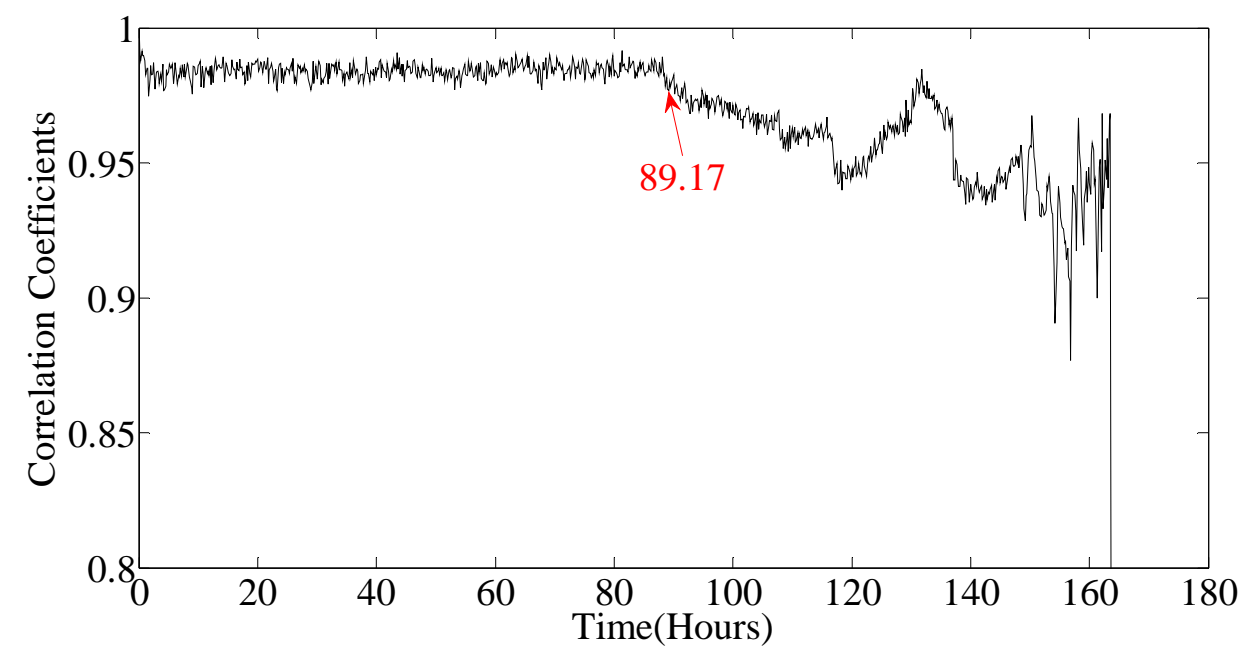

Fig. 5 Monitoring results for the rolling bearing using the SLA-based method

\section{Summary}

This paper introduced SLA to study rolling bearing vibration data. By SLA, the rank index sequence can be obtained. Then, the correlation coefficient between the rank index sequence for the starting moment and that for another moment was define as the measure for detecting a change of running conditions of rolling bearings. Afterwards, this paper proposed a novel method for condition monitoring of rolling bearings using SLA. Application to condition monitoring of the realistic rolling bearing indicated that the proposed method can detect a change of operating conditions of rolling bearings earlier than the other two methods. Therefore, the proposed method seemingly outperforms the traditional methods in condition monitoring of rolling bearings.

\section{References}

[1] W. He, Y. Zi, B. Chen, F. Wu, Z. He, Automatic fault feature extraction of mechanical anomaly on induction motor bearing using ensemble super-wavelet transform, Mech. Syst. Signal Process., 54 (2015) 457-480.

[2] X. Fan, M.J. Zuo, Gearbox fault detection using Hilbert and wavelet packet transform, Mech. Syst. Signal Process., 20 (2006) 966-982.

[3] N.E. Huang, Z. Shen, S.R. Long, M.C. Wu, H.H. Shih, Q. Zheng, N.-C. Yen, C.C. Tung, H.H. Liu, The empirical mode decomposition and the Hilbert spectrum for nonlinear and non-stationary time series analysis, Proc. R. Soc. Lond. A, 454 (1998) 903-995.

[4] Z.H. Wu, N.E. Huang, Ensemble empirical mode decomposition: a noise-assisted data analysis method, Adv. Adapt. Data. Anal., 1 (2009) 1-41.

[5] A.C.-C. Yang, S.-S. Hseu, H.-W. Yien, A.L. Goldberger, C.-K. Peng, Linguistic analysis of the human heartbeat using frequency and rank order statistics, Phys. Rev. Lett., 90 (2003) 108103.

[6] H. Qiu, J. Lee, J. Lin, G. Yu, Wavelet filter-based weak signature detection method and its application on rolling element bearing prognostics, Journal of sound and vibration, 289 (2006) 1066-1090. 